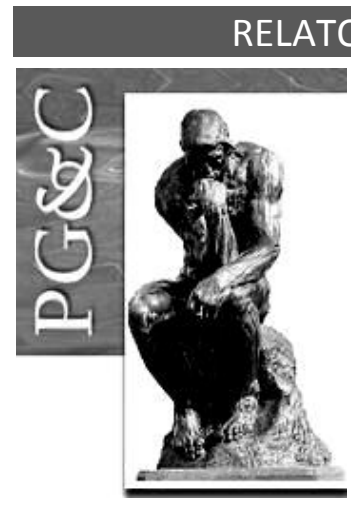

\title{
A GESTÃO DO CONHECIMENTO EM UMA INSTITUIÇÃO DE ENSINO PRIVADA ${ }^{1}$
}

\author{
Jefferson Rodrigues Pereira \\ Mestre em Administração pela Faculdade Novos Horizontes, Brasil. \\ E-mail: jeffersonrodrigues@live.com \\ Leonardo Benedito Oliveira Rezende \\ Mestre em Administração pela Faculdade Novos Horizontes, Brasil. \\ E-mail: leorezende100@gmail.com \\ Aleixina Maria Lopes Andalécio \\ Doutora em Ciência da Informação pela Universidade Federal de Minas \\ Gerais, Brasil. Professora da Faculdade Novos Horizontes, Brasil. \\ E-mail: aleixina.andalecio@unihorizontes.br

\section{Caissa Veloso e Sousa} \\ Doutora em Administração de Empresas pela Universidade Federal de \\ Minas Gerais, Brasil. Professora da Faculdade Novos Horizontes, Brasil. \\ E-mail: caissaveloso@yahoo.com.br \\ Eliane Bragança de Matos \\ Doutora em Administração pela Universidade Federal de Minas Gerais, \\ Brasil. Professora do Centro Federal de Educação Tecnológica - CEFET, \\ Minas Gerais, Brasil. \\ E-mail: elianebraganca@gmail.com
}

\begin{abstract}
Resumo
Na sociedade contemporânea, o conhecimento e o capital humano assumem papel de destaque no ambiente organizacional, sendo o conhecimento um recurso econômico significante. Diante desse contexto, o presente artigo relata pesquisa desenvolvida com o objetivo de identificar e analisar a percepção de funcionários de uma instituição particular de ensino infantil, fundamental e médio, localizada na região metropolitana de Belo Horizonte/MG, acerca das práticas de gestão do conhecimento, tendo por base o modelo proposto por Terra (2005). Para tal, realizou-se um estudo de caso descritivo, de abordagem quantitativa. Para a análise dos dados adotou-se como método a análise fatorial exploratória. Dentre os resultados alcançados, destaca-se a presença de bidimensionalidade em três dentre as sete dimensões da Gestão do Conhecimento propostas por Terra (2005). Importa salientar que a maioria das médias entre as variáveis analisadas tendiam a se posicionar com uma concordância negativa acerca da informação oferecida pela variável. Tal fato leva a inferir que, no ambiente onde a pesquisa foi desenvolvida, a gestão do conhecimento ainda não é uma questão fundamentada, navegando na contra mão do que é proposto pela teoria.
\end{abstract}

Palavras-chave: Gestão do Conhecimento. Estratégia. Conhecimento Organizacional.

\footnotetext{
${ }^{1}$ Versão reformulada e aprofundada de artigo publicado no V Encontro de Administração da Informação (EnADI), promovido pela Associação Nacional de Pós-Graduação em Administração, sob responsabilidade da Divisão Acadêmica de Administração da Informação (ADI). O evento ocorreu no período de 21 a 23 de junho de 2015 em Brasília, Distrito Federal, Brasil.
}

Perspectivas em Gestão \& Conhecimento, João Pessoa, v. 6, n. 2, p. 113-133, jul./dez. 2016. http://periodicos.ufpb.br/ojs2/index.php/pgc. ISSN: 2236-417X. Publicação sob Licença (cc) EY-NC-ND 


\title{
KNOWLEDGE MANAGEMENT IN A PRIVATE EDUCATION INSTITUTION
}

\begin{abstract}
In contemporary society, knowledge and human capital play a crucial role in the organizational environment, considering the knowledge a significant economic resource. In this context, the present paper reports research conducted in order to identify and analyze the perception of employees of a private school, located in the metropolitan region of Belo Horizonte / MG, about the knowledge management practices, based on the model proposed by Terra (2005). We performed a descriptive case study with a quantitative approach. For the data analysis we used the exploratory factorial analysis. The results shows the presence of two-dimensionality in three of the seven dimensions of Knowledge Management proposed by Terra (2005). It should be noted that most average among the variables analyzed tended to be positioned with a negative agreement about the information being provided by variable. This fact leads to the inference that the environment where the research was developed, knowledge management is not a reasoned question, surfing the hand against the theory.
\end{abstract}

Keywords: Knowledge Management. Strategy. Organizational knowledge.

\section{INTRODUÇÃO}

A sociedade contemporânea possui como uma de suas principais características a mudança contínua e rápida. Nesse cenário, Terra $(1999,2005)$ sublinha a importância do conhecimento e do capital humano, bem como sua gestão, no ambiente organizacional. Em complemento, Carbone et al. (2005) destacam que esses recursos devem ser analisados estrategicamente, uma vez que são fontes seguras de vantagem competitiva.

Segundo Drucker (1999), em uma sociedade pós-capitalista, à qual ele se refere como sociedade do conhecimento, o conhecimento transcende o valor do capital e do trabalho, sendo ele o recurso econômico de maior significância nesse contexto.

Para Turthil (1990), o conhecimento é um processo interno de assimilação e análise das informações recebidas, que pode variar de indivíduo para indivíduo. Segundo Sveiby (1988), o conhecimento refere-se a uma "capacidade de agir" e não pode ser destacado do ambiente.

Choo $(2003$, p. 27) ressalta que, na concepção atual da administração e da teoria organizacional, "a criação e o uso da informação desempenham um papel estratégico no crescimento e na capacidade de adaptação da empresa". Nesse sentido, importa salientar que, no âmbito organizacional, a criação do conhecimento ocorre de dentro para fora das empresas, com o intuito de solucionar problemas de seu ambiente (NONAKA; TAKEUCHI, 1997).

Nesse contexto, Goulart e Angeloni (2009) ressaltam que o conhecimento assume o papel de fator crítico, tanto de sucesso, quanto de insucesso empresarial. Essas autoras destacam a Gestão do Conhecimento como importante aliada da estratégia da organização, dado que, por meio dela, torna-se possível a criação de ações formais no que tange a criação e compartilhamento de conhecimento entre as pessoas.

Para Oliveira (2002), a Gestão do Conhecimento pode ser compreendida como um processo intencional, articulado e sistematizado, cujo principal objetivo é a geração, disseminação, codificação e apropriação de conhecimentos, em busca de melhoria contínua. Ainda segundo o autor, ela auxilia nos processos organizacionais, uma vez que permite a organização, além de gerar conhecimento, armazená-lo, distribuí-lo e utilizá-lo da maneira mais eficaz e eficiente possível.

Davenport (1998, p. 12) destaca a importância de os gestores terem "uma perspectiva holística, que possa assimilar alterações repentinas no mundo dos negócios e adaptar-se às

Perspectivas em Gestão \& Conhecimento, João Pessoa, v. 6, n. 2, p. 113-133, jul./dez. 2016 
sempre mutantes realidades sociais". Nesse ambiente um desafio das empresas é descobrir como estimular a disseminação do conhecimento e a aprendizagem organizacional, bem como gerenciar estrategicamente esse processo, permitindo que uma organização ofereça um produto ou serviço de maior valor agregado a seus clientes, adquirindo vantagem competitiva e diferenciando-se dos concorrentes (OLIVEIRA et al., 2001; SANTOS; VALENTIM, 2014).

Nesse contexto, reporta-se às colocações de Terra (2005), segundo o qual, uma das grandes importâncias da gestão do conhecimento organizacional é tornar mais mensurável o conhecimento disperso no interior da mesma. Nesse sentido, o autor propõe um modelo conceitual baseando-se em sete dimensões, a saber: (I) Visão estratégica; (II) Cultura organizacional; (III) Estrutura organizacional; (IV) Políticas de recursos humanos; (V) Sistemas de informação; (VI) Mensuração de resultados; e, (VII) Aprendizado com o ambiente.

Mediante a esse cenário, construiu-se a seguinte problemática de estudo: como os funcionários de uma instituição de ensino percebem as práticas de gestão do conhecimento por ela empreendidas?

Como objetivo geral buscou-se identificar e analisar a percepção dos funcionários de uma instituição particular de ensino infantil, fundamental e médio, acerca das práticas de gestão do conhecimento, tendo por base o modelo proposto por Terra (2005).

0 presente artigo está dividido em cinco seções. A primeira compreende esta introdução, na qual são apresentados o tema, problemática e objetivo de pesquisa. A segunda seção apresenta o referencial teórico, que oferece suporte à discussão ora proposta, e a terceira descreve a metodologia adotada na construção da pesquisa. Segue-se a quarta seção, na qual os dados são apresentados e analisados. Por fim, encontram-se as considerações finais do estudo, bem como suas limitações e sugestões de futuras pesquisas, e as referências utilizadas.

\section{REFERENCIAL TEÓRICO}

Esta seção apresenta o referencial teórico que embasou a pesquisa, contemplando o tema gestão do conhecimento e descrevendo as sete dimensões da gestão do conhecimento, as quais foram a base para a coleta e análise dos dados.

\subsection{Gestão do conhecimento}

Apesar de os primeiros estudos acerca da importância do conhecimento no desenvolvimento organizacional datarem da década de 1970, quando o termo gestão do conhecimento foi cunhado por Henry (1974), para referir-se a políticas públicas para produção, acessibilidade, divulgação e utilização da informação, ressalta-se pouca representatividade acadêmica do tema até o final da década de 1980 (SANTOS NETTO, 2004). Segundo Nonaka e Takeuchi (1997), os estudos sobre esse assunto se intensificaram no início da década de 1990.

A acentuada representatividade acadêmica da temática na década de 1990 se justifica em função das constantes transformações da sociedade da informação, atreladas à necessidade de ações inteligentes, por parte das organizações, frente ao cenário de alterações nos padrões competitivos (NONAKA; TAKEUCHI, 1997; SANTOS et al., 2001; FREIRE et al., 2012; SÁ et al., 2013). Neste contexto, surge a demanda para que as empresas se modifiquem e façam uma análise criteriosa de suas bases informacionais, seus conhecimentos e de sua tecnologia da informação (TI), no sentido de atingirem a excelência na gestão do conhecimento (PARSONS, 1983; TAPSCOTT, 1997; NONAKA; TAKEUCHI, 1997).

Sveiby (1998) ressalta que o tema transcende a ideia, até então difundida, de um modismo de eficiência operacional, tornando-se parte integrante da estratégia da organização. Nesse aspecto é possível inferir que a relevância da gestão do conhecimento no ambiente

Perspectivas em Gestão \& Conhecimento, João Pessoa, v. 6, n. 2, p. 113-133, jul./dez. 2016 
empresarial, e no meio acadêmico, se dá em função da forte relação existente entre o mercado, cada vez mais competitivo, e as necessidades das organizações em atenderem as expectativas dos clientes (SANTOS NETTO, 2004). Corroborando este posicionamento, Costa (2013) salienta que a temática é, na atualidade, fonte de grandes debates, uma vez que sua principal função está em apoiar as empresas em ações para melhorar os resultados e agregar valores aos clientes.

Oliveira $(2002$, p. 83$)$ ressalta que a gestão do conhecimento pode ser vista como

[...] um processo sistemático, articulado e intencional, apoiado na geração, codificação, disseminação e apropriação de conhecimentos, com o propósito de atingir a "excelência organizacional", que consiste em cumprir seus propósitos da melhor forma possível, encantando seus clientes (internos e externos), engajada em um processo interminável de melhoria contínua.

Tal afirmação permite inferir que apenas possuir dados em grande quantidade não constitui, por si só, um mecanismo que conduza a ganhos competitivos, esses devem ser trabalhados. Nesse sentido, sublinha-se que a grande quantidade de dados disponíveis, associada à velocidade de sua circulação, culminou na necessidade de uma seleção criteriosa e sistematizada desses dados no ambiente organizacional (CARBONE et al., 2005). A partir de então, os dados se tornam passíveis de serem transformados em conhecimento, possibilitando assim, que as pessoas possam analisá-los, selecioná-los de acordo com a necessidade específica da organização a qual se insere e, a partir de então, tornar possível a elaboração de estratégias que possam contribuir para o desenvolvimento da empresa (TAPSCOTT, 1997; FREIRE et al., 2012; COSTA, 2013; SANTOS; VALENTIM, 2014).

Portanto, essa sistematização transcende o que é explícito, constituindo-se em algo que combine o conhecimento explícito com o tácito e esses entre si, a partir do ambiente no qual estão inseridos. Atualmente, o conhecimento é visto como o bem de maior valor de uma organização e o indivíduo o ativo mais importante nesse contexto, dado que todo conhecimento é oriundo das pesquisas e experiências humanas (CARBONE et al., 2005; CARDOSO, 2008; SÁ et al., 2013). Assim, autores como Parsons (1983) e Goulart e Angeloni (2009), apontam que a gestão do conhecimento, além de se destacar no meio acadêmico, tem gerado interesse empresarial, devido à necessidade de se administrar o ser humano (e o ambiente no qual este está inserido, dadas suas interações), o único ser pensante na organização e, portanto, importante fator estratégico na tentativa de se alcançar os objetivos da empresa.

Terra (2005) enfatiza que a gestão do conhecimento está diretamente associada às práticas gerenciais e as etapas do aprendizado individual e compartilhado. Segundo esse autor, o fenômeno ainda se associa à coordenação dos esforços em níveis organizacionais e pessoais, às normas formais e informais e aos fatores estratégicos e operacionais.

Nesse contexto, o capital intelectual emerge como um importante ativo do ambiente corporativo. Na visão de Stewart (1998), o capital intelectual pode ser compreendido como todo o conhecimento existente no interior de uma organização, ou seja, é constituído pela matéria intelectual, como a informação, a propriedade intelectual, o conhecimento e experiências que podem ser utilizadas no sentido de gerar riqueza e aumentar o nível de competitividade da empresa.

Alvares, Baptista e Araújo Júnior (2010) ressaltam que as empresas, atualmente, estão imersas na era do conhecimento e saber lidar com o capital intelectual da empresa se tornou algo imprescindível. Segundo esses autores, nesse ambiente, a informação se tornou determinante para, além do processo de produção de conhecimento, a competitividade. 
Em consonância com este posicionamento, Santos Netto (2004) salienta que o principal impacto do conhecimento nas organizações é a competitividade. Para ele, a era da informação alterou o quadro social, dando origem aos novos saberes e a sociedade do conhecimento, o que, por sua vez, criou, nas organizações a necessidade de redefinir o perfil do indivíduo enquanto trabalhador.

Mediante a esse cenário ressalta-se o posicionamento de Terra (1999), segundo o qual, tanto na organização quanto na sociedade, o conhecimento deve se estender a todos os níveis. Segundo esse autor, o não acesso ao conhecimento por determinada parte da população é um assunto sério demais para ser deixado de lado. Para esse autor, a questão do conhecimento abrange todos os segmentos e, somente em um contexto de um conhecimento abrangente a todos os níveis e áreas sociais, uma organização ou sociedade pode se tornar uma grande potência, desenvolvida em diversos dos seus setores.

Nesse aspecto destaca-se que tanto o conhecimento tácito, ou seja, aquele que não é facilmente quantificado e está presente na "mente" do indivíduo, quanto o conhecimento explícito, que compreende aquele que já foi formalizado e está disponível para acesso a outros indivíduos, são importantes para a geração e disseminação do conhecimento organizacional, sendo que o conhecimento tácito, dado o seu caráter subjetivo, é de mais difícil compartilhamento, pois depende tanto de quem o compartilha quanto de quem o recebe, para maior assimilação (TAKEUCHI; NONAKA, 2008).

Destaca-se ainda que a geração de conhecimento não está restrita as ações gerenciais, mas o seu gerenciamento pode proporcionar melhores resultados em termos dos resultados pretendidos (TAKEUCHI; NONAKA, 2008). Considerado esses aspectos Terra (2005) salienta que, no processo de gestão do conhecimento, o conhecimento tácito, ou seja, o conhecimento subjetivo está atrelado a um conjunto de práticas de gerenciamento ligado aos modos de conversão de conhecimento. Nesse sentido, o autor propõe um modelo conceitual de gestão do conhecimento baseado em sete dimensões, o qual é descrito na próxima seção.

\subsection{As sete dimensões da gestão do conhecimento}

O modelo conceitual de gestão do conhecimento proposto por Terra (2005) busca tornar mais mensurável os diversos tipos de conhecimentos existentes no interior da organização e baseia-se em sete dimensões. Tal modelo está representado na Figura 1, e as dimensões envolvidas são:

1. Visão estratégica (alta administração): esta dimensão tem por objetivo analisar a relação que se constrói entre conhecimento organizacional e estratégia corporativa;

2. Cultura Organizacional: nesta dimensão, a cultura é analisada como fator facilitador ou dificultador no processo de criação e disseminação de conhecimento no ambiente organizacional;

3. Estrutura organizacional: visa analisar o modelo de gestão mais adequado no que tange as práticas de gestão do conhecimento;

4. Política de recursos humanos: tem como foco as políticas de gestão de pessoas da organização, analisando como ela pode atuar no sentido de geração, difusão e armazenamento de conhecimento na organização;

5. Sistemas de informação: avalia as bases de dados, incluindo a tecnologia da informação, que, por meio de sua evolução, modificou a estrutura de comunicação no interior das empresas;

6. Mensuração de resultados: analisa as práticas adotadas pela organização em relação às diferentes dimensões do capital intelectual; 
7. Aprendizado com o ambiente: esta dimensão busca analisar a competência organizacional no que se refere ao seu aprendizado com o ambiente, que se dá, principalmente, por meio do relacionamento estabelecido com os clientes e alianças estratégicas.

Figura 1 - Gestão do conhecimento: planos e dimensões

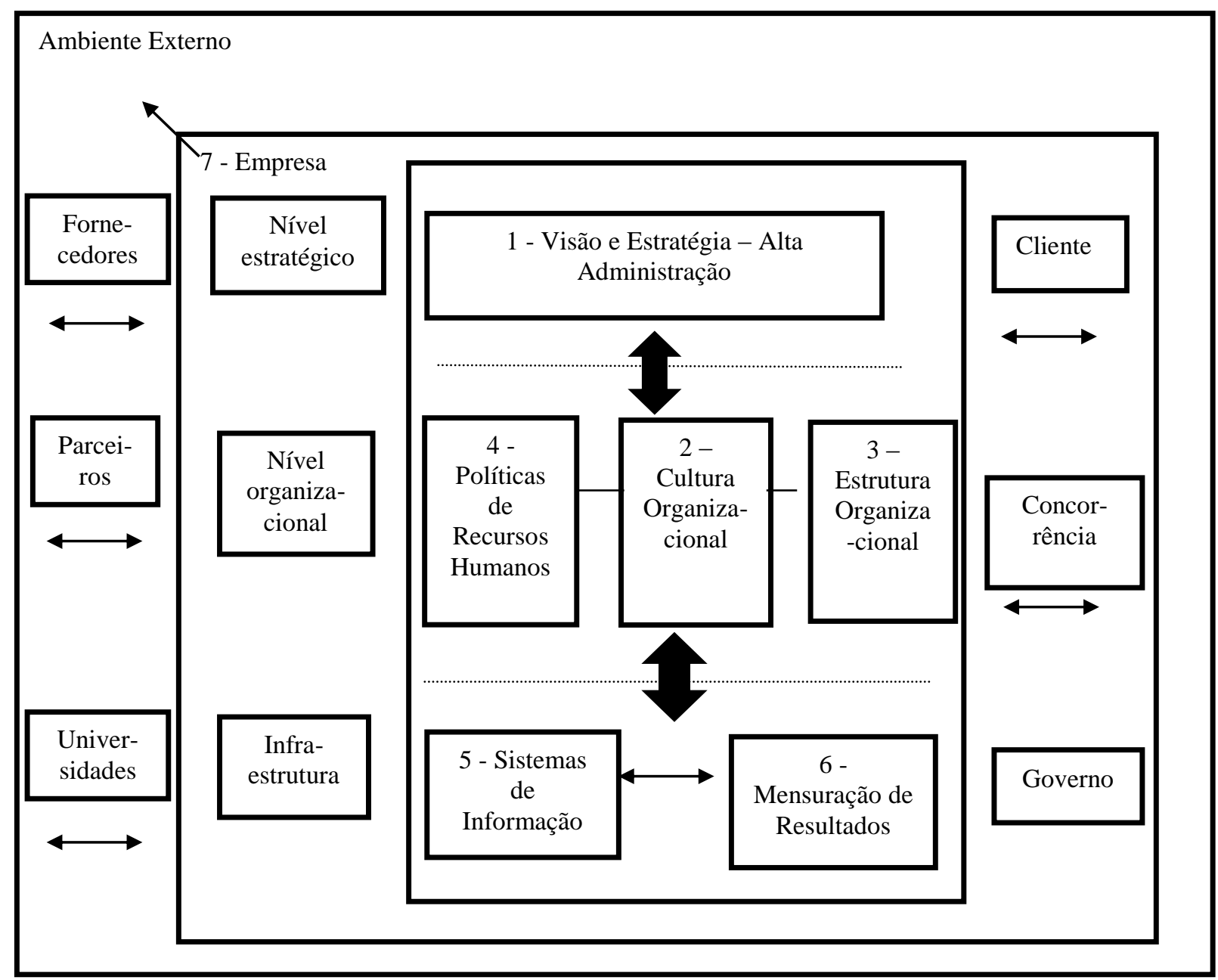

Fonte: Terra (2005, p. 86)

Essas sete dimensões da gestão do conhecimento propostas por Terra (2005) foram utilizadas como embasamento para a construção do instrumento de pesquisa e para a análise dos dados da pesquisa relatada neste artigo.

\section{METODOLOGIA}

A pesquisa aqui apresentada adotou a abordagem quantitativa e, quanto aos fins, se classifica como descritiva, uma vez que se propôs a analisar características de determinada população sem, contudo, oferecer explicação para o fenômeno (GIL, 2002). Quanto aos meios, a pesquisa se caracteriza como um estudo de caso, que, segundo Vergara (2007), busca analisar, por meio de uma investigação empírica, determinado fenômeno em seu ambiente real de observação.

O estudo foi realizado em uma instituição de ensino privada, localizada na Região Metropolitana de Belo Horizonte. A organização pesquisada possui 89 pessoas registradas no

Perspectivas em Gestão \& Conhecimento, João Pessoa, v. 6, n. 2, p. 113-133, jul./dez. 2016 
quadro de funcionários. $O$ público alvo da escola compreende alunos das classes $A$ e $B$, do ensino infantil ao ensino médio. A escolha da instituição se deu por acessibilidade.

$O$ instrumento de coleta de dados utilizado foi um questionário estruturado em escala Likert de cinco pontos, sendo utilizado o modelo validado por Terra (2005). A escala proposta pelo autor contém sete dimensões, somando 36 variáveis. O Quadro 1 apresenta as dimensões, os indicadores e as variáveis analisadas.

Quadro 1 - Relação de Dimensões, variáveis e indicadores

\begin{tabular}{|c|c|c|}
\hline Dimensão & Variáveis & Indicador \\
\hline \multirow{3}{*}{$\begin{array}{l}\text { Estratégia e } \\
\text { Alta Direção } \\
\quad \text { (EAD) }\end{array}$} & $\begin{array}{l}\text { Existe elevado nível de consenso sobre quais são as competências } \\
\text { essenciais (core competences) da instituição, ou seja, todos sabem quais } \\
\text { são os pontos fortes da instituição em termos de habilidades e } \\
\text { competências. }\end{array}$ & EAD1 \\
\hline & $\begin{array}{l}\text { A macroestratégia da Instituição é comunicada, amplamente, para todos } \\
\text { os níveis organizacionais. }\end{array}$ & EAD2 \\
\hline & $\begin{array}{l}\text { A alta administração estabelece, frequentemente, metas desafiadoras e } \\
\text { um sentido de urgência para a mudança da realidade em direção a uma } \\
\text { visão estabelecida. }\end{array}$ & EAD3 \\
\hline \multirow{10}{*}{$\begin{array}{l}\text { Cultura } \\
\text { Organizacional } \\
\text { (CO) }\end{array}$} & $\begin{array}{l}\text { A missão e os valores da instituição são promovidos, de forma } \\
\text { consistente, por meio de atos simbólicos e ações. }\end{array}$ & $\mathrm{CO} 4$ \\
\hline & $\begin{array}{l}\text { Há um elevado sentimento de confiança entre instituição e } \\
\text { colaboradores; existe, de maneira geral, um grande orgulho em } \\
\text { trabalhar para a instituição. }\end{array}$ & CO5 \\
\hline & $\begin{array}{l}\text { As pessoas desenvolvem seus trabalhos com foco também no médio e } \\
\text { longo prazo. }\end{array}$ & CO6 \\
\hline & Estimula-se a experimentação. Há liberdade para tentar e falhar. & $\mathrm{CO7}$ \\
\hline & $\begin{array}{l}\text { As pessoas são autênticas e deixam evidente aquilo que conhecem e } \\
\text { também o que não conhecem. }\end{array}$ & $\mathrm{CO8}$ \\
\hline & $\begin{array}{l}\text { As pessoas estão preocupadas com toda a instituição e não apenas com } \\
\text { sua área de trabalho, ou seja, buscam uma otimização conjunta. }\end{array}$ & CO9 \\
\hline & $\begin{array}{l}\text { As pessoas reconhecem que o tempo é um recurso importante para o } \\
\text { processo de inovação. }\end{array}$ & $\mathrm{CO} 10$ \\
\hline & Novas ideias são valorizadas. Há permissão para discutir ideias "bobas". & CO11 \\
\hline & As realizações importantes são comemoradas. & $\mathrm{CO} 12$ \\
\hline & Há grande tolerância para piadas e humor. & CO13 \\
\hline \multirow{6}{*}{$\begin{array}{l}\text { Organização e } \\
\text { Processos de } \\
\text { Trabalho } \\
\text { (OPT) }\end{array}$} & $\begin{array}{l}\text { Há um uso constante de equipes multidisciplinares e formais que se } \\
\text { sobrepõem à estrutura formal tradicional e hierárquica. }\end{array}$ & OPT14 \\
\hline & $\begin{array}{l}\text { Há um uso constante de equipes "ad-hoc" ou temporárias, com grande } \\
\text { autonomia, totalmente dedicadas a projetos inovadores. }\end{array}$ & OPT15 \\
\hline & $\begin{array}{l}\text { Pequenas reorganizações ocorrem com frequência, de forma natural, } \\
\text { para se adaptar às demandas do ambiente competitivo. }\end{array}$ & OPT16 \\
\hline & $\begin{array}{l}\text { Realizam-se, com frequência, reuniões informais, fora do local de } \\
\text { trabalho, para a realização de brainstorms. }\end{array}$ & OPT17 \\
\hline & $\begin{array}{l}\text { Os lay-outs são conducentes à troca informal de informação (uso de } \\
\text { espaços abertos e salas de reunião). São poucos os símbolos de status e } \\
\text { hierárquicos. }\end{array}$ & OPT18 \\
\hline & $\begin{array}{l}\text { As decisões são tomadas no nível mais baixo possível. O processo } \\
\text { decisório é ágil; a burocracia é mínima. }\end{array}$ & OPT19 \\
\hline Políticas e & O processo de seleção é bastante rigoroso. & PP20 \\
\hline
\end{tabular}

Perspectivas em Gestão \& Conhecimento, João Pessoa, v. 6, n. 2, p. 113-133, jul./dez. 2016 


\begin{tabular}{|c|c|c|}
\hline \multirow{7}{*}{$\begin{array}{l}\text { práticas para a } \\
\text { Administração } \\
\text { de Recursos } \\
\text { Humanos (PP) }\end{array}$} & $\begin{array}{l}\text { Há uma busca de diversidade (personalidades, experiências, cultura, } \\
\text { educação formal e outros) e aumento da criatividade por meio do } \\
\text { recrutamento. }\end{array}$ & PP21 \\
\hline & $\begin{array}{l}\text { O planejamento de carreira busca dotar os colaboradores com } \\
\text { diferentes perspectivas e experiências. }\end{array}$ & PP22 \\
\hline & $\begin{array}{l}\text { As descrições das responsabilidades dos cargos são, em geral, bastante } \\
\text { abrangentes. }\end{array}$ & PP23 \\
\hline & $\begin{array}{l}\text { Há um elevado investimento e incentivo ao treinamento e } \\
\text { desenvolvimento profissional e pessoal dos funcionários. Estimulam-se } \\
\text { treinamentos que levam ao autoconhecimento. }\end{array}$ & PP24 \\
\hline & $\begin{array}{l}\text { Estimula-se o aprendizado por meio da ampliação dos contatos e } \\
\text { interações com outras pessoas de dentro e fora da instituição. }\end{array}$ & PP25 \\
\hline & $\begin{array}{l}\text { O treinamento está associado às necessidades da área imediata de } \\
\text { trabalho do colaborador e/ou às necessidades estratégicas da } \\
\text { instituição. }\end{array}$ & PP26 \\
\hline & $\begin{array}{l}\text { Há um baixo turnover (número de pessoas que se demitem ou são } \\
\text { demitidas) na instituição, em comparação a outras instituições do } \\
\text { mesmo setor. }\end{array}$ & PP27 \\
\hline \multirow{3}{*}{$\begin{array}{l}\text { Sistemas de } \\
\text { Informação e } \\
\text { Comunicação } \\
\text { (SIC) }\end{array}$} & $\begin{array}{l}\text { A comunicação é eficiente em todos os sentidos (de cima para baixo, de } \\
\text { baixo para cima e entre áreas distintas). }\end{array}$ & $\mathrm{SIC} 28$ \\
\hline & $\begin{array}{l}\text { As informações são compartilhadas. Existe amplo acesso, por parte de } \\
\text { todos os funcionários, à base de dados e conhecimento da instituição. }\end{array}$ & SIC29 \\
\hline & $\begin{array}{l}\text { Há grande disciplina, eficiência e incentivo para o registro do } \\
\text { conhecimento e "know-how" existentes na instituição. }\end{array}$ & SIC30 \\
\hline \multirow{2}{*}{$\begin{array}{l}\text { Mensuração } \\
\text { de Resultados } \\
\text { (MR) }\end{array}$} & $\begin{array}{l}\text { Existe uma grande preocupação em medir resultados sob várias } \\
\text { perspectivas (financeiras, operacionais, estratégicas, aquisição de } \\
\text { conhecimento). }\end{array}$ & MR31 \\
\hline & Os resultados são amplamente divulgados internamente. & MR32 \\
\hline \multirow{4}{*}{$\begin{array}{l}\text { Aprendizado } \\
\quad \text { com o } \\
\text { Ambiente (AA) }\end{array}$} & $\begin{array}{l}\text { A instituição aprende muito com seus clientes. Existem vários } \\
\text { mecanismos formais e informais que possibilitam à instituição aprender } \\
\text { com seus clientes e outros parceiros. }\end{array}$ & AA33 \\
\hline & $\begin{array}{l}\text { A instituição tem habilidade na gestão de parcerias com outras } \\
\text { empresas. }\end{array}$ & AA34 \\
\hline & $\begin{array}{l}\text { A instituição tem habilidade na gestão de parcerias com outras } \\
\text { Universidades e Institutos de Pesquisa (contratação de pesquisa } \\
\text { externa). }\end{array}$ & AA35 \\
\hline & $\begin{array}{l}\text { A decisão de realizar alianças está, frequentemente, relacionada a } \\
\text { decisões estratégicas e de aprendizado importantes. Os colaboradores } \\
\text { da instituição percebem, muito claramente, esse objetivo de } \\
\text { aprendizado. }\end{array}$ & AA36 \\
\hline
\end{tabular}

Fonte: Adaptado de Terra (2005)

Antes da aplicação do questionário foi realizado um pré-teste com 20 funcionários durante o mês de fevereivo de 2015 para verificar questões relacionadas à clareza e à objetividade das variáveis propostas. Nesse processo não foi identificado nenhum fator de impedimento acerca da aplicabilidade do instrumento.

Findada a etapa de pré-teste iniciou-se a fase de coleta dos dados, que ocorreu durante os meses de fevereiro e março de 2015, na qual o questionário foi aplicado em funcionários da instituição que ocupavam cargos administrativos, de gestão e docentes. Participaram deste estudo 60 funcionários selecionados pelo critério de acessibilidade. 
Os dados foram analisados por meio da análise multivariada de dados, sendo que se realizou a Análise Fatorial Exploratória (AFE) para cada dimensão proposta por Terra (2005). A AFE pode ser compreendida como uma técnica multivariada, cujo objetivo está em identificar uma estrutura subjacente de uma matriz de dados e determinar a natureza e o número dos fatores (as variáveis latentes) que possam representar da maneira mais fiel possível determinado conjunto de variáveis observadas (BROWN, 2006). Hair Junior et al. (2005), complementam que o ideal, no caso da análise multivariada, é que se tenha um mínimo de cinco observações para cada variável analisada, sendo o ideal que se atinja dez observações por variável (HAIR JUNIOR et al., 2005; FIELD, 2009).

Ressalta-se que o mínimo de variáveis sugeridas pelos autores não foi atingido, sendo coletados 1,81 questionários por variável. Entretanto, destaca-se que $67 \%$ da população (60 pessoas) responderam ao questionário e, ainda, que não se pretende apresentar aqui uma perspectiva de generalização de resultados, mas sim analisar o fenômeno a luz do mercado educacional. Para cada dimensão foram analisados:

- Escore fatorial: compreende os escores obtidos por meio da combinação linear das variáveis (HAIR JUNIOR et al., 2005);

- Alfa de Cronbach (geral e se item excluído): este índice é uma medida de confiabilidade que mede a consistência das variáveis que compõem a amostra analisada, sendo desejável valores mínimos acima de 0,70 , para as ciências sociais (HAIR JUNIOR et al., 2005). Em complemento, Field (2009) ressalta que, ao passo que o número de itens em uma escala aumenta, o alfa tende a aumentar, fato que implica que em alguns casos um valor do alfa de Cronbach mais baixo pode ser visto como satisfatório;

- Kaiser-Meyer-Olkin (KMO): medida de adequacidade da amostra. De acordo com Field (2009), para o KMO, os valores que figuram acima de 0,70 são considerados "regulares" e os que se encontram acima de 0,80 "muito bons" e o limite inferior para aceitação do modelo deve ficar acima de 0,50;

- Teste de esfericidade de Bartlett: que tem como objetivo avaliar a hipótese de as variáveis não serem correlacionadas com na população (MALHOTRA, 2001). Em complemento, Sampaio (2012) destaca que tal teste verifica se os dados contêm suficiente evidência que comprovem a hipótese de que a matriz de correlação não é uma matriz identidade. Norusis (1999) ressalta que a utilização da análise fatorial está condicionada a rejeitar a hipótese de que a matriz de correlação de uma população é uma identidade;

- Nível de significância estatística (Sig): trata-se de uma medida estimada que busca aferir o grau em que o resultado alcançado é "verdadeiro" (SAMPAIO, 2012);

- Variância extraída: trata-se de uma medida de consistência complementar interna. Este índice objetiva-se em medir a quantidade geral de variância dos indicadores explicada pela variável latente e, seus valores devem figurar acima de 0,50 (HAIR JUNIOR et al., 2005).

\section{APRESENTAÇÃO E ANÁLISE DOS DADOS}

Na presente seção, os dados da pesquisa são apresentados e analisados por meio da análise fatorial exploratória, tendo por base as sete dimensões da gestão do conhecimento propostas por Terra (2005).

Perspectivas em Gestão \& Conhecimento, João Pessoa, v. 6, n. 2, p. 113-133, jul./dez. 2016 


\subsection{Estratégia e alta direção}

Esta dimensão buscou analisar a relação existente entre o conhecimento organizacional e a estratégia da organização e a Tabela 1 apresenta os valores encontrados, os quais indicam que o construto estratégia e alta direção apresentou unidimensionalidade formada pelos indicadores EAD1, AED2 e EAD3.

Tabela 1 - Estratégia e alta direção

\begin{tabular}{|c|c|c|c|c|}
\hline Construto & Indicadores & Escore Fatorial & Alfa & Alfa se item deletado \\
\hline \multirow{3}{*}{ Estratégia e alta direção } & EAD1 & 0,689 & \multirow{3}{*}{0,585} & 0,539 \\
\hline & EAD2 & 0,813 & & 0,350 \\
\hline & EAD3 & 0,715 & & 0,519 \\
\hline KMO & & & & 0,598 \\
\hline Bartlett's Test & & & & 17,687 \\
\hline Sig & & & & 0,001 \\
\hline Variância extraída & & & & $54,880 \%$ \\
\hline
\end{tabular}

Fonte: Dados da pesquisa (2015)

Quanto aos parâmetros do construto, o KMO ficou abaixo do valor desejável, porém acima do limite inferior de acordo com Field (2009). A confiabilidade do construto se posicionou abaixo do valor mínimo aceito, com um alfa de Cronbach de 0,585. Nesse aspecto, salienta-se que, segundo Field (2009) este fato pode ser explicado dado o reduzido número de itens da escala.

Os demais parâmetros de avaliação de unidimensionalidade de construto obtiveram índices que atendem aos parâmetros encontrados na literatura.

\subsection{Cultura organizacional}

Esta dimensão pautou suas análises na cultura organizacional, enquanto facilitadora ou dificultadora da criação e disseminação do conhecimento na organização. Inicialmente, foi identificada a presença de bidimensionalidade no construto. A partir disso, foi feita novamente a análise fatorial, cujos resultados são mostrados nas Tabela 2 e Tabela 3.

Tabela 2 - Cultura organizacional (Subconstruto 1 - SCO1)

\begin{tabular}{|c|c|c|c|c|}
\hline Construto & Indicadores & Escore Fatorial & Alfa & Alfa se item deletado \\
\hline \multirow{6}{*}{ Cultura Organizacional } & $\mathrm{CO} 4$ & 0,624 & \multirow{6}{*}{0,784} & 0,770 \\
\hline & $\mathrm{CO5}$ & 0,748 & & 0,739 \\
\hline & $\mathrm{CO} 7$ & 0,760 & & 0,734 \\
\hline & Co11 & 0,696 & & 0,753 \\
\hline & CO12 & 0,753 & & 0,732 \\
\hline & CO13 & 0,579 & & 0,778 \\
\hline $\mathrm{KMO}$ & & & & 0,782 \\
\hline Bartlett's Test & & & & 86,843 \\
\hline Sig & & & & 0,000 \\
\hline Variância extraída & & & & $48,538 \%$ \\
\hline
\end{tabular}

Fonte: Dados da pesquisa (2015)

Como apresentado na Tabela 2, o subconstruto 1 (SCO1) atingiu resultados satisfatórios em quase todos seus índices, a exceção foi a variância extraída que se fixou em $48,538 \%$.

Perspectivas em Gestão \& Conhecimento, João Pessoa, v. 6, n. 2, p. 113-133, jul./dez. 2016 
O segundo subconstruto desta dimensão (SCO2), inicialmente era composto pelos indicadores CO6, CO8, CO9 e CO10. Os indicadores CO6 (As pessoas desenvolvem seus trabalhos com foco também no médio e longo prazo) e CO8 (As pessoas são autênticas e deixam evidente aquilo que conhecem e também o que não conhecem) foram excluídos, pois apresentaram alfas de Cronbach para os indicadores superiores ao alfa do subconstruto, alcançando-se os resultados expostos na Tabela 3.

Tabela 3 - Cultura organizacional (Subconstruto 2 - SCO2)

\begin{tabular}{|c|c|c|c|c|}
\hline Construto & Indicadores & Escore Fatorial & Alfa & Alfa se item deletado \\
\hline \multirow{2}{*}{ Cultura Organizacional } & $\mathrm{CO9}$ & 0,902 & \multirow{2}{*}{0,768} & * \\
\hline & CO10 & 0,902 & & * \\
\hline KMO & & & & 0,500 \\
\hline Bartlett's Test & & & & 28,710 \\
\hline Sig & & & & 0,000 \\
\hline Variância extraída & & & & $81,347 \%$ \\
\hline
\end{tabular}

Fonte: Dados da pesquisa (2015)

O subconstruto composto pelos indicadores $\mathrm{CO}$ e $\mathrm{CO} 10$ apresentou valores de alfa de Cronbach, teste de esfericidade de Bartlett e variância extraída coerentes com o descrito na literatura. Contudo, o valor do KMO se fixou em 0,500, limite inferior para aceitação do modelo (FIELD, 2009).

\subsection{Organização e processos de trabalho}

De acordo com Terra (2005), tal dimensão tem como objetivo analisar o modelo de gestão mais adequado no que tange as práticas de gestão do conhecimento.

Os resultados desta dimensão, a exemplo do ocorrido na dimensão Cultura Organizacional, apresentou bidimensionalidade. Na construção do primeiro subconstruto (SOPT1) excluíram-se os indicadores OPT14 (Há um uso constante de equipes multidisciplinares e formais que se sobrepõem à estrutura formal tradicional e hierárquica) e OPT16 (Pequenas reorganizações ocorrem com frequência, de forma natural, para se adaptar às demandas do ambiente competitivo), por apresentarem alfas de Cronbach superiores ao do subconstruto. A Tabela 4 apresenta os resultados do subconstruto.

Tabela 4 - Organização e processos de trabalho (Subconstruto 1 - SOPT1)

\begin{tabular}{|c|c|c|c|c|}
\hline Construto & Indicadores & Escore Fatorial & Alfa & Alfa se item deletado \\
\hline \multirow{2}{*}{$\begin{array}{c}\text { Organização e Processo de } \\
\text { Trabalho }\end{array}$} & OPT18 & 0,878 & \multirow{2}{*}{0,701} & \\
\hline & OPT19 & 0,878 & & * \\
\hline KMO & & & & 0,500 \\
\hline Bartlett's Test & & & & 19,894 \\
\hline & & & & 0,000 \\
\hline Variância extraída & & & & $77,040 \%$ \\
\hline
\end{tabular}

Fonte: Dados da pesquisa (2015)

Este subconstruto apresentou valores satisfatórios para quase todos os índices em questão. A exceção foi o KMO, que atingiu um valor de 0,500, se fixando no limite inferior da aceitação do modelo, segundo Field (2009).

O segundo subconstruto (SOPT2) foi composto pelos indicadores OPT15 e OPT17 e seus resultados são apresentados na Tabela 5.

Perspectivas em Gestão \& Conhecimento, João Pessoa, v. 6, n. 2, p. 113-133, jul./dez. 2016 
Tabela 5 - Organização e processos de trabalho (Subconstruto 2 - SOPT2)

\begin{tabular}{|c|c|c|c|c|}
\hline Construto & Indicadores & Escore Fatorial & Alfa & Alfa se item deletado \\
\hline \multirow{2}{*}{$\begin{array}{c}\text { Organização e Processo de } \\
\text { Trabalho }\end{array}$} & OPT15 & 0,818 & \multirow{2}{*}{0,507} & * \\
\hline & OPT17 & 0,818 & & * \\
\hline $\mathrm{KMO}$ & & & & 0,500 \\
\hline Bartlett's Test & & & & 7,055 \\
\hline Sig & & & & 0,008 \\
\hline Variância extraída & & & & $66,991 \%$ \\
\hline
\end{tabular}

Fonte: Dados da pesquisa (2015)

A constituição deste subconstruto atingiu valores baixos para o alfa de Cronbach $(0,507)$ e KMO $(0,500)$. Ressalta-se que, apesar de baixo, para as ciências sociais esse valor é aceitável (SAMPAIO, 2012). Os demais indicadores apresentaram valores satisfatórios.

\subsection{Políticas e práticas para a administração de recursos humanos}

Terra (2005) destaca que esta dimensão tem como foco as políticas de gestão de pessoas da organização, analisando sua atuação no que tange a gestão do conhecimento.

Este construto também se dividiu em dois fatores, apresentando bidimensionalidade. Importa destacar que, na redução de fatores da primeira dimensão, excluíram-se os indicadores PP20 (O processo de seleção é bastante rigoroso) e PP27 (Há um baixo turnover número de pessoas que se demitem ou são demitidas - na instituição, em comparação a outras instituições do mesmo setor), uma vez que esses indicadores apresentaram valores para o alfa de Cronbach superior ao alfa do construto. O subconstruto 1 (SPP1), composto pelos indicadores PP24, PP25 e PP26, é descrito na Tabela 6.

Tabela 6 - Políticas e práticas para a administração de recursos humanos (Subconstruto 1 - SPP1)

\begin{tabular}{|c|c|c|c|c|}
\hline Construto & Indicadores & Escore Fatorial & Alfa & Alfa se item deletado \\
\hline \multirow{3}{*}{$\begin{array}{c}\text { Políticas Práticas para a } \\
\text { Administração de Recursos } \\
\text { Humanos }\end{array}$} & PP24 & 0,881 & \multirow{3}{*}{0,856} & 0,800 \\
\hline & PP25 & 0,873 & & 0,814 \\
\hline & PP26 & 0,892 & & 0,780 \\
\hline KMO & & & & 0,733 \\
\hline Bartlett's Test & & & & 77,537 \\
\hline Sig & & & & 0,000 \\
\hline Variância extraída & & & & 77,791 \\
\hline
\end{tabular}

Fonte: Dados da pesquisa (2015)

$\mathrm{Na}$ constituição dos valores expostos pela Tabela 6, destaca-se que todos os indicadores atenderam aos parâmetros expostos na literatura.

Na constituição do segundo subconstruto da presente dimensão (SPP2), excluiu-se, inicialmente, o indicador PP23 (As descrições das responsabilidades dos cargos são, em geral, bastante abrangentes), dado que a exclusão do indicador fez com que aumentasse o alfa de Cronbach do construto. Os índices calculados para o subconstruto SPP2 são expostos na Tabela 7.

Tabela 7 - Políticas e práticas para a administração de recursos humanos (Subconstruto 2 - SPP2)

\begin{tabular}{c|c|r|r|r}
\hline \hline Construto & Indicadores & Escore Fatorial & Alfa & Alfa se item deletado \\
\hline $\begin{array}{c}\text { Políticas Práticas para a } \\
\text { Administração de Recursos } \\
\text { Humanos }\end{array}$ & PP21 & 0,916 & \multirow{2}{*}{0,808} & $*$ \\
\cline { 2 - 3 } & PP22 & 0,916 & & $*$ \\
\hline KMO & \multicolumn{3}{|c}{0,500} \\
\hline \hline
\end{tabular}

Perspectivas em Gestão \& Conhecimento, João Pessoa, v. 6, n. 2, p. 113-133, jul./dez. 2016 


\begin{tabular}{l|r}
\hline Bartlett's Test & 35,335 \\
Sig & 0,000 \\
Variância extraída & $83,878 \%$ \\
\hline \hline
\end{tabular}

Fonte: Dados da pesquisa (2015)

No que tange aos resultados alcançados neste subconstruto, destaca-se que o único índice que não atingiu o valor parametrizado pela literatura foi o $\mathrm{KMO}$, que se fixou no limite inferior da aceitação do modelo (FIELD, 2009).

\subsection{Sistemas de informação e comunicação}

Baseando-se em Terra (2005), esta dimensão tem como objetivo analisar as bases de dados e a tecnologia da informação da organização. Este construto apresentou unidimensionalidade e os índices alcançados por meio da análise fatorial são expostos na Tabela 8:

Tabela 8 - Sistemas de informação e comunicação

\begin{tabular}{|c|c|c|c|c|}
\hline Construto & Indicadores & Escore Fatorial & Alfa & Alfa se item deletado \\
\hline \multirow{2}{*}{$\begin{array}{c}\text { Sistemas de Informação e } \\
\text { Comunicação }\end{array}$} & SIC28 & 0,905 & \multirow{2}{*}{0,775} & $*$ \\
\hline & SIC29 & 0,905 & & $*$ \\
\hline KMO & & & & 0,500 \\
\hline Bartlett's Test & & & & 29,937 \\
\hline Sig & & & & 0,000 \\
\hline Variância extraída & & & & $81,854 \%$ \\
\hline
\end{tabular}

Fonte: Dados da pesquisa (2015)

Destaca-se que, na redução fatorial da presente dimensão, excluiu-se o indicador SIC30 (Há grande disciplina, eficiência e incentivo para o registro do conhecimento e "know how" existentes na instituição), fato que permitiu o aumento do valor do alfa de Cronbach do construto. Na sequência, a análise dos indicadores SIC28 e SIC29 do construto atendeu aos critérios apontados pela literatura, com exceção do KMO, que ficou abaixo do recomendado, se fixando em 0,500 , limite inferior da validação do modelo, conforme exposto por Field (2009).

\subsection{Mensuração de resultados}

Este construto tem como objetivo criar uma análise das práticas adotadas pela organização no que tange ao capital intelectual e as suas dimensões (TERRA, 2005). Ele apresentou unidimensionalidade e seus resultados são expostos na Tabela 9.

Tabela 9 - Mensuração de resultados

\begin{tabular}{|c|c|c|c|c|}
\hline Construto & Indicadores & Escore Fatorial & Alfa & Alfa se item deletado \\
\hline \multirow{2}{*}{$\begin{array}{l}\text { Mensuração de } \\
\text { Resultados }\end{array}$} & MR31 & 0,811 & \multirow{2}{*}{0,480} & $*$ \\
\hline & MR32 & 0,811 & & * \\
\hline $\mathrm{KMO}$ & & & & 0,500 \\
\hline Bartlett's Test & & & & 6,068 \\
\hline Sig & & & & 0,014 \\
\hline Variância extraída & & & & $65,823 \%$ \\
\hline
\end{tabular}

Fonte: Dados da pesquisa (2015)

Perspectivas em Gestão \& Conhecimento, João Pessoa, v. 6, n. 2, p. 113-133, jul./dez. 2016 
Tendo por base os parâmetros apresentados na literatura, destaca-se que o construto mensuração de resultados apresentou o índice KMO fixado em 0,500, limite inferior para aceitação do modelo (FIELD, 2009). Identificou-se ainda um valor abaixo dos parâmetros literários para o alfa de Cronbach, contudo, o teste de esfericidade de Bartlett e a variância extraída atingiram o índice proposto por Hair Junior et al. (2005).

\subsection{Aprendizado com o ambiente}

A última dimensão da gestão do conhecimento proposta por Terra (2005) visa analisar a competência organizacional e seu elo com o ambiente, buscando compreender como se configura o aprendizado neste ambiente. Os índices apontados na redução fatorial deste construto são apresentados na Tabela 10.

Tabela 10 - Aprendizado com o ambiente

\begin{tabular}{l|c|r|r|r}
\hline \hline \multicolumn{1}{c|}{ Construto } & Indicadores & Escore Fatorial & Alfa & Alfa se item deletado \\
\hline \multirow{3}{*}{ Aprendizado com o Ambiente } & AA33 & 0,648 & & 0,685 \\
\cline { 2 - 3 } & AA34 & 0,689 & \multirow{2}{*}{0,704} & 0,663 \\
\cline { 2 - 3 } & AA35 & 0,811 & 0,574 \\
\cline { 2 - 3 } & AA36 & 0,753 & & 0,625 \\
\hline KMO & & & 0,621 \\
Bartlett's Test & & & 49,020 \\
Sig & & & 0,000 \\
Variância extraída & & & $52,964 \%$ \\
\hline \hline
\end{tabular}

Fonte: Dados da pesquisa (2015)

Conforme mostrado na Tabela 10, esse construto apresentou unidimensionalidade formada pelos quatro indicadores (AA33, AA34, AA35 e AA36). Os parâmetros recomendados pela literatura também foram atendidos, com exceção do KMO, que se fixou em 0,621. A confiabilidade do construto também foi aceitável, com um alfa de Cronbach de 0,704.

\subsection{Análise descritiva dos dados}

A presente seção apresenta a análise descritiva dos dados, baseando-se na média do indicador, uma medida de posição, desvio padrão e coeficiente de variação de Pearson, que são medidas de dispersão (MALHOTRA, 2001).

De acordo com Pearson (1914), o coeficiente de variação (C.V.), é uma medida relativa e seu cálculo é obtido a partir do quociente entre o desvio padrão e a média amostral, na qual sua análise está baseada nas seguintes relações:

- Se C.V. < 15\%, a dispersão é baixa;

- Se $15 \%<$ C.V. $<30 \%$, a dispersão é média;

- Se C.V. > 30\%, a dispersão é elevada.

A Tabela 11 apresenta os resultados da estatística descritiva obtida em cada constructo, sendo destacadas, em cada um deles, a variável com menor média e aquela com maior média em negrito. 
Tabela 11 - Estatística descritiva dos dados

\begin{tabular}{|c|c|c|c|}
\hline Indicador & Média & Desvio Padrão & C.V. Pearson \\
\hline EAD1 & 3,200 & 1,054 & 32,952 \\
\hline EAD2 & 2,600 & 1,330 & 51,162 \\
\hline EAD3 & 3,183 & 0,965 & 30,326 \\
\hline $\mathrm{CO} 4$ & 2,983 & 1,255 & 42,080 \\
\hline $\mathrm{CO} 5$ & 3,233 & 1,140 & 35,271 \\
\hline CO6 & 3,183 & 1,157 & 36,347 \\
\hline $\mathrm{CO} 7$ & 2,683 & 1,214 & 45,251 \\
\hline $\mathrm{CO} 8$ & 2,617 & 0,940 & 35,941 \\
\hline CO9 & 2,650 & 1,087 & 41,000 \\
\hline CO10 & 3,450 & 0,982 & 28,452 \\
\hline Co11 & 2,967 & 1,301 & 43,869 \\
\hline CO12 & 2,783 & 1,403 & 50,418 \\
\hline $\mathrm{CO} 13$ & 2,867 & 1,157 & 40,348 \\
\hline OPT14 & 2,783 & 1,027 & 36,885 \\
\hline OPT15 & 2,383 & 1,106 & 46,410 \\
\hline OPT16 & 3,200 & 1,205 & 37,641 \\
\hline OPT17 & 1,917 & 1,169 & 60,974 \\
\hline OPT18 & 2,433 & 1,095 & 44,998 \\
\hline OPT19 & 1,983 & 1,186 & 59,797 \\
\hline PP20 & 2,767 & 1,212 & 43,823 \\
\hline PP21 & 3,117 & 1,136 & 36,459 \\
\hline PP22 & 2,933 & 1,133 & 38,624 \\
\hline PP23 & 3,300 & 1,266 & 38,371 \\
\hline PP24 & 2,467 & 1,255 & 50,880 \\
\hline PP25 & 2,533 & 1,112 & 43,889 \\
\hline PP26 & 2,867 & 1,214 & 42,343 \\
\hline PP27 & 3,000 & 1,426 & 47,538 \\
\hline SIC28 & 2,567 & 1,280 & 49,887 \\
\hline SIC29 & 2,417 & 1,139 & 47,143 \\
\hline SIC30 & 2,967 & 1,104 & 37,219 \\
\hline MR31 & 3,617 & 1,136 & 31,419 \\
\hline MR32 & 2,867 & 1,081 & 37,706 \\
\hline AA33 & 3,233 & 1,095 & 33,864 \\
\hline AA34 & 3,350 & 1,117 & 33,352 \\
\hline AA35 & 2,933 & 1,287 & 43,877 \\
\hline AA36 & 2,983 & 1,214 & 40,700 \\
\hline
\end{tabular}

Fonte: Dados da pesquisa (2015)

O construto estratégia e alta direção (EAD), composto pelos indicadores EAD1, EAD2 e EAD3, apresentou dispersão elevada dos dados. Identificou-se ainda, por parte dos respondentes, uma baixa percepção de eficácia no que se refere à comunicação da macroestratégia da organização, ao passo que, com uma dispersão média, a maioria dos respondentes concorda parcialmente que os pontos fortes da instituição (habilidade e competências) são disseminados na empresa.

O segundo construto analisado, cultura organizacional (CO), é formado pelos indicadores de $\mathrm{CO} 4$ a $\mathrm{CO} 13$, e apresentou um índice dispersivo alto. A maioria da amostra pesquisada aponta que, na organização, a autenticidade das pessoas não é demonstrada, ou seja, elas não deixam evidenciar aquilo que sabem ou não. Por outro lado, com baixa dispersão, os respondentes concordam que o tempo é um recurso importante no processo de inovação. 
Já no construto organização e processo de trabalho (OPT), constituído pelos indicadores OPT14 a OPT19, identificou-se um elevado nível de dispersão dos dados. A amostra tende a concordar que, frequentemente, ocorrem reorganizações no sentido de adaptar-se às demandas externas do ambiente. Contudo, com o maior coeficiente de variação de Pearson das variáveis apresentadas, aponta-se que não são realizadas reuniões informais fora do local de trabalho.

$\mathrm{Na}$ análise do construto política e práticas (PP), composto pelos indicadores PP20 a PP27, identificou-se uma dispersão alta, indicando que, neste contexto, as descrições acerca das responsabilidades dos cargos são abrangentes. Apesar do elevado indice de dispersão amostral, identificou-se, ainda, uma baixa percepção dos respondentes a respeito de investimentos, por parte da organização, em treinamentos e desenvolvimento de pessoas.

O construto sistema de informação e comunicação (SIC), formado pelos indicadores SIC28, SIC29 e SIC30, apesar de apresentar uma grande dispersão amostral, apontou que as informações acerca da organização objeto de análise são mantidas em sigilo. Importa destacar, ainda, que a média de maior valor do construto não atinge o nível de concordância parcial de que, há incentivo para registro do conhecimento produzido na organização.

No construto mensuração de resultados (MR), composto pelos indicadores MR31 e MR32, identificou-se, com o segundo menor coeficiente de variação de Pearson e a maior média amostral, que a principal preocupação da instituição está em medir seus resultados sob várias perspectivas. Contudo, verificou-se que os resultados alcançados não são divulgados internamente.

Por fim, a análise do último construto da gestão do conhecimento proposto por Terra (2005), aprendizado com o ambiente (AA), formado pelos indicadores AA33, AA34, AA35 e AA36, indicou que, com um nível de dispersão elevado, a amostra tendeu a concordar parcialmente que a instituição tem uma habilidade na gestão de parcerias, compondo redes com outras organizações. Apesar disso, a menor média do construto apontou que essas parcerias, geralmente, não ocorrem com universidades ou institutos de pesquisa, ou seja, não realizam-se contratações de pesquisas externas para auxiliá-la no processo de gestão.

\subsection{Análise da correlação de Pearson}

Segundo Malhotra (2001), a correlação linear de Pearson é um indicador adimensional e possui variação entre -1 e 1 . Quando há um alinhamento perfeito dos dados em uma reta com declividade negativa, o coeficiente de Pearson é igual a -1 (correlação linear negativa perfeita), ao passo que, se os dados se encontram alinhados perfeitamente em uma reta com declividade positiva ocorre a chamada correlação linear positiva perfeita, atingindo um valor para o coeficiente de Pearson igual a 1 (FIGUEIREDO FILHO; SILVA JUNIOR, 2009).

De acordo com Garson (2009), a correlação de Pearson ( $r$ ) pode ser entendida como uma forma de medir a associação bivariada do grau de correlação (relacionamento) entre duas variáveis e, para Moore (2007), esse indicador busca mensurar o grau de relação linear entre duas variáveis, bem como sua direção (positiva ou negativa). Acerca desse coeficiente, destaca-se que, segundo Franzblau (1958):

- Se $r=0$, não existe relação linear entre as duas variáveis;

- Se $|r|<0,20$, a correlação é negligenciável;

- Se $0,20<|r|<0,40$, a correlação é fraca;

- Se $0,40<|r|<0,60$, a correlação é moderada;

- Se $0,60<|r|<0,80$, a correlação é forte;

- Se $|r|>0,80$, a correlação é muito forte. 
Tendo por base esses valores de referência do coeficiente de correlação de Pearson (FRANZBLAU, 1958), a Tabela 12 apresenta os valores dos coeficientes de correlação entre os construtos e subconstrutos que foram objeto de análise nesta pesquisa, sendo destacados em negrito os valores que indicam a existência de correlação forte.

Tabela 12 - Matriz de coeficiente de correlação de Pearson

\begin{tabular}{c|c|c|c|c|c|c|c|c|c}
\hline \hline & EAD & SCO1 & SCO2 & SOPT1 & SOPT2 & SPP1 & SPP2 & SIC & MR \\
\hline SCO1 & $\mathbf{0 , 6 4 9}$ & & & & & & & & \\
SCO2 & 0,387 & 0,386 & & & & & & & \\
SOPT1 & 0,330 & 0,406 & 0,411 & & & & & & \\
SOPT2 & 0,202 & 0,196 & 0,025 & 0,311 & & & & & \\
SPP1 & 0,149 & 0,162 & 0,178 & 0,333 & 0,497 & & & & \\
SPP2 & 0,522 & 0,323 & 0,326 & 0,243 & 0,268 & 0,461 & & & \\
SIC & 0,544 & $\mathbf{0 , 6 2 2}$ & 0,403 & 0,574 & 0,296 & 0,294 & 0,246 & & \\
MR & 0,382 & 0,347 & 0,314 & 0,287 & 0,239 & 0,340 & 0,261 & 0,402 & \\
AA & 0,512 & 0,366 & 0,475 & 0,375 & 0,395 & 0,534 & 0,447 & 0,395 & 0,530 \\
\hline \hline
\end{tabular}

Fonte: Dados da pesquisa (2015)

De acordo com esses dados, apenas duas correlações entre os construtos podem ser consideradas fortes, ou seja, a correlação entre SCO1 (subconstruto cultura organizacional 1) e EAD (estratégia e alta direção), cujo $r=0,649$, e a existente entre SIC (sistemas de informação e comunicação) e SCO1 (subconstruto cultura organizacional 1) com $r=0,622$. Quatorze correlações podem ser consideradas de intensidade moderada, vinte e quatro são de intensidade fraca e cinco correlações são negligenciáveis, de acordo com Franzblau (1958).

No que tange a correlação de forte intensidade observada entre os construtos SCO1 e EAD, destaca-se o posicionamento de Davenport (1998), segundo quem o ambiente estratégico da alta direção possui uma forte relação com a cultura organizacional. Para esse autor, a organização deve dispender uma atenção especial para o elo formado entre a estratégia de negócios, a cultura organizacional e a orientação dos recursos humanos, uma vez que este refletirá os componentes do ambiente informacional: "Assim, a estratégia de negócios, por exemplo, irá influenciar a estratégia da informação e vice-versa" (DAVENPORT, 1998, p. 55).

Outro ponto importante a ser salientado é a forte correlação que é possível observar entre a cultura organizacional e os sistemas de informação e comunicação ( $r=0,622)$, permitindo identificar uma relação de influência mútua entre estes dois construtos.

Para se compreender a relação entre estes dois construtos, deve-se, inicialmente, considerar a grande influência exercida pelos fatores humanos nos sistemas de informação, descrita por Amorim e Tomaél (2012). Ainda, segundo essas autoras, os sistemas de informação são "condicionados pelo nível de envolvimento e de entendimento das pessoas com relação ao que é proposto, ou seja, mudanças de procedimentos advindos de sistemas informatizados" (AMORIM; TOMAÉL, 2012, p. 64).

Ainda nesse contexto, Davenport, Prusak e Eccles (1998) destacam a importância de a cultura organizacional ser receptiva ao processo de gestão da informação, salientando que "o fluxo de informações não torna a cultura organizacional menos hierárquica e mais aberta; ao contrário, as culturas democráticas tornam possíveis os fluxos democráticos de informações" (DAVENPORT; PRUSAK; ECCLES, 1998, p.173). 


\section{CONSIDERAÇÕES FINAIS}

A partir da década de 1990, a gestão do conhecimento, enquanto temática, adentrou com maior representatividade no cenário acadêmico e corporativo. Isso em função de um processo de intensificação de mudanças na sociedade da informação, associado à necessidade de as organizações se tornarem cada vez mais competitivas (NONAKA; TAKEUCHI, 1997; SANTOS et al., 2001).

Mediante esse cenário, o presente artigo relata pesquisa desenvolvida com o objetivo de analisar a gestão do conhecimento, enquanto processo, em um ambiente escolar. Para tal optou-se por utilizar o modelo das sete dimensões da gestão do conhecimento validado por Terra (2005). Ao se analisar a unidimensionalidade para cada dimensão proposta no modelo, identificou-se, a partir da análise fatorial, que quatro dimensões (Estratégia e Alta Direção, Sistemas de Informação e Comunicação, Mensuração de Resultados e Aprendizado com o Ambiente) apresentaram apenas um construto extraído, com elevada variância explicada. Isso reflete que as variáveis desse construto explicam o construto que representa a respectiva dimensão.

Contudo, identificou-se ainda que, em três das sete dimensões (Cultura Organizacional, Organização e Processos de Trabalho e Políticas e Práticas para a Administração de Recursos Humanos), a presença de dois fatores, dando origem dois subconstrutos cada. Dada essa ocorrência, são observados os seguintes aspectos: a) o tamanho da amostra pode ter reflexos na caracterização do modelo, o que poderia ser melhor visualizado analisando-se outras escolas com o mesmo perfil e aumentando o tamanho da amostra; b) apesar de o tamanho da amostra não atender aos critérios mínimos estabelecidos por autores como Hair Junior et al. (2005) e Field (2009), esse representa mais de $60 \%$ da população e, portanto, pode ser um indicativo de que o modelo, para esse ramos de atividade, precise ser revisto para melhor adequação.

Por meio da análise da correlação de Pearson, observaram-se apenas duas correlações classificadas como fortes, tendo por base a literatura. Sendo elas a relação existente entre o subconstruto cultura organizacional 1 (SCO1) e o construto estratégia e alta direção (EAD), atingindo um $r=0,649$ e a correlação que se estabelece entre o construto sistema de informação e comunicação (SIC) e o subconstruto cultura organizacional 1 (SCO1), índice que atingiu o valor de $r=0,622$.

Por fim, importa salientar que, para o caso do presente estudo, a maioria das médias entre as variáveis analisadas tendiam a se posicionar com uma concordância negativa acerca da informação oferecida pela variável. Tal fato leva a inferir que, no ambiente onde a presente pesquisa foi desenvolvida, a gestão do conhecimento ainda não é uma questão fundamentada, contrariando o previsto na teoria pertinente.

Como limitações do presente estudo identifica-se a amostra reduzida na qual o estudo se balizou. Portanto, para futuros estudos, sugere-se a replicação da pesquisa com amostras maiores. Sugere-se também o desenvolvimento de pesquisas de abordagens qualitativas, a fim de que os resultados aqui alcançados sejam explorados em maior nível de profundidade. Ainda, acredita-se ser importante testar o modelo em outras instituições de ensino, o que permitiria identificar possíveis necessidades de adaptação (ou não) do modelo.

\section{REFERÊNCIAS}

ALVARES, Lillian; BAPTISTA, Sofia G.; ARAÚJO JÚNIOR, Rogério Henrique. Gestão do Conhecimento. Brasília: Universidade de Brasília, Faculdade de Ciência da Informação, 2010. Disponível em: http://www.alvarestech.com/lillian/Projeto/Modulo3/AreasDelnterseccao.pdf. Acesso em: 20 mar. 2015.

Perspectivas em Gestão \& Conhecimento, João Pessoa, v. 6, n. 2, p. 113-133, jul./dez. 2016 
AMORIM, Fabiana R. B.; TOMAÉL, Maria Inês. A influência da cultura organizacional na utilização de sistemas de informações em organizações públicas. Informação@Profissões, Londrina, v. 1, n. 1/2, p. 56-76, 2012.

BERMEJO, Paulo Henrique $S$. et al. Uma Visão do Desenvolvimento de Estratégias de TI Utilizando a Gestão do Conhecimento. Revista de Ciências da Administração, p. 139-155, 2014.

BROWN, T. A. Confirmatory factor analysis for applied research. New York: The Guilford Press, 2006.

CARBONE, Pedro P. et al. Gestão do capital Intelectual: a mensuração de ativos intangíveis. In: 3, p. 101-148. Gestão por competências e gestão do conhecimento. Rio de Janeiro: FGV, 2005. Cap.

CARDOSO, Leonor. Gestão do conhecimento e competitividade organizacional: um modelo estrutural. Comportamento Organizacional e Gestão, v. 13, n. 2, p. 191-211, out. 2008. Disponível em: http://www.scielo.oces.mctes.pt/scielo.php?script=sci arttext\& pid= S087296622007000200004\&lng=pt\&nrm=iso. Acesso em: 20 mar. 2015.

CHOO, Chun W. A organização do conhecimento: uma visão holística de como as organizações usam a informação. In: . A organização do conhecimento: como as organizações usam a informação para criar significado, construir conhecimento e tomar decisões. São Paulo: SENAC, 2003.

COSTA, Heloísa F. A. Práticas da Gestão do Conhecimento: avaliação de Curso de Administração das Instituições de Ensino Superior Privadas de Minas Gerais baseada no Exame Nacional de Desempenho do Estudante. 2013. 99 f. Dissertação (Mestrado em Administração) - Faculdade de Ciências Empresariais, Fundação Mineira de Educação e Cultura de Minas Gerais, Belo Horizonte, 2013.

DAVENPORT, Thomas $\mathrm{H}$. Ecologia da informação: por que só a tecnologia não basta para o sucesso na era da informação. São Paulo: Futura, 1998.

DAVENPORT, Thomas H.; PRUSAK, Laurence; ECCLES, Robert G. Política da informação. In: KLEIN, David. A gestão estratégica do capital intelectual. Rio de Janeiro: Qualitymark, 1998. p. 151-182.

DRUCKER, Peter F. Desafios gerenciais para o século XXI. São Paulo: Pioneira, 1999.

FIELD, Andy. Descobrindo a estatística usando o SPSS. Porto Alegre: Artmed, 2009.

FIGUEIREDO FILHO, Dalson B.; SILVA JUNIOR, José A. Desvendando os Mistérios do Coeficiente de Correlação de Pearson (r). Revista Política Hoje, v. 18, n. 1, 2010.

FRANZBLAU, Abraham N. A primer of statistics for non-statisticians. Nova York: Harcourt, Brace and World, 1958.

FREIRE, Patrícia S. et al. Memória organizacional e seu papel na gestão do conhecimento. Revista de Ciências da Administração, v. 14, n. 33, p. 41-51, 2012.

GARSON, David G. Statnotes: Topics in Multivariate Analysis, 2009. Disponível em: http://faculty.chass.ncsu.edu/garson/PA765/statnote.htm. Acesso em: 20 mar. 2015.

GIL, Antônio C. Como elaborar projetos de pesquisa. 4. ed. São Paulo: Atlas, 2002. 
GOULART, Elizane; ANGELONI, Maria Terezinha. O compartilhamento do conhecimento em uma instituição de ensino superior. Revista Brasileira de Docência, Ensino e Pesquisa em Administração, Edição Especial, v. 1, n. 2, p. 59-83, 2009.

HAIR JR, Joseph F. et al. Análise Multivaridada de dados. Porto Alegre: Bookman, 2005.

HENRY, Nicholas L. Knowledge management: a new concern for public administration. Public Administration Review, Washington, v.34, n.3, p. 189-196, 1974.

MALHOTRA, Naresh. Pesquisa de marketing: uma orientação aplicada. Porto Alegre: Bookman, 2001.

MOORE, David S. The Basic Practice of Statistics. New York, Freeman, 2007.

NONAKA, Ikujiro; TAKEUCHI, Hirotaka. Criação do conhecimento na empresa: como as empresas japonesas geram a dinâmica da inovação. Rio de Janeiro: Campus, 1997.

. Gestão do conhecimento. São Paulo: Bookman, 2008.

NORUSIS, Marija J. SPSS 9.0 Guide to data analysis. Upper Saddle River, New Jersey: Prentice Hall, 1999.

OLIVEIRA, Luiz C. A. et al. A tecnologia da informação como instrumento do posicionamento estratégico. In: ENCONTRO DA ASSOCIAÇÃO NACIONAL DE PÓS-GRADUAÇÃO E PESQUISA EM ADMINISTRAÇÃO, 25., 2001, Campinas. Anais... Rio de Janeiro: ANPAD 2001.

OLIVEIRA, Luciel H. Gestão do Conhecimento como fonte de vantagem competitiva sustentável para as instituições de ensino superior (IES). Revista Acadêmica da FACECA - RAF, Varginha, v. 1, n. 2, jan./jul. 2002.

PARSONS, Gregory L. Information technology: a new competitive weapon. Sloan Management Review, v. 1, n. 25, p. 3-14, 1983.

PEARSON, Karl. "On the probability that two independent distributions of frequency are really samples of the same population, with special reference to recent work on the identily of trypanosome strains". Biometrika, v.10, n.1, p.85-143, 1914.

SÁ, Fabiano B. et al. Práticas de gestão do conhecimento: Um estudo em organizações mineiras. Perspectivas em Gestão \& Conhecimento, v. 3, n. 1, p. 114-131, 2013.

SAMPAIO, Danilo O. Intenção de compra e consumo de alimentos orgânicos: um estudo sobre crenças, atributos e grupos de referência. 2012. 219 f. Tese (Doutorado em Administração) Centro de Pós Graduação e Pesquisa em Administração, Universidade Federal de Minas Gerais, Belo Horizonte, 2012.

SANTOS, Antônio R. et al. (Orgs.). Gestão do conhecimento: uma experiência para o sucesso empresarial. Curitiba: Champagnat, 2001.

SANTOS NETTO, João Paulo. Gestão do conhecimento e competitividade. In: SANTOS, Silvio A. dos; PASQUALE, Perrotti Pietrangelo (Org.). Fronteiras da administração I: teorias, aplicações e tendências. Campinas: Akademika, 2004. Cap. 3, p. 49-65.

SANTOS, Cássia D.; VALENTIM, Marta Lígia P. Gestão de fluxos de informação em ambiente organizacional. Perspectivas em Gestão \& Conhecimento, João Pessoa, v. 4, n. 2, p. 19-33, 2014.

STEWART, Thomas A. Capital intelectual: a nova vantagem competitiva das empresas. Rio de Janeiro: Campus, 1998.

Perspectivas em Gestão \& Conhecimento, João Pessoa, v. 6, n. 2, p. 113-133, jul./dez. 2016 
SVEIBY, Karl E. A nova riqueza das organizações: gerenciando e avaliando patrimônios de conhecimento. 5. ed. Rio de Janeiro: Campus, 1998.

TAPSCOTT, Don. Economia digital. São Paulo: Makron Books, 1997.

TERRA, José Cláudio C. Gestão do Conhecimento: aspectos conceituais e estudo exploratório sobre as práticas de empresas brasileiras. 1999. $311 \mathrm{f}$. Tese (Doutorado em Engenharia de Produção) - Escola Politécnica da Universidade de São Paulo, São Paulo, 1999.

Gestão do Conhecimento: o grande desafio empresarial; inclui o modelo das sete dimensões da gestão do conhecimento. 5. ed. Rio de Janeiro: Elsevier, 2005.

TURTHIL, Steven G. Knowledge engineering: concepts and practices for knowledge-based system. Blue Ridge Summit, PA: Tab Books, 1990.

VERGARA Sylvia C. Projetos e relatórios de pesquisa em Administração. 8. ed. São Paulo: Atlas, 2007. 